\title{
Pigment deposition in the reticuloendothelial system after fat emulsion infusion
}

\author{
J. H. PASSWELL, R. DAVID, D. KATZNELSON, and B. E. COHEN \\ From the Departments of Paediatrics and Pathology, Chaim Sheba Medical Centre, and Tel-Aviv University \\ Medical School, Israel
}

\begin{abstract}
Passwell, J. H., David, R., Katznelson, D., and Cohen, B. E. (1976). Archives of Disease in Childhood, 51, 366. Pigment deposition in the reticuloendothelial system after fat emulsion infusion. In 2 infants who had received Intralipid fat emulsion as part of a total parenteral hyperalimentation regimen, a pigmented material was deposited in the macrophages of their reticuloendothelial systems. The histochemical characteristics of this pigment were similar to those seen after experimental infusion of fat emulsions. The possible implications of this finding and its effect on host resistance are briefly considered.
\end{abstract}

Parenteral hyperalimentation has now been widely adopted in the supportive care of infants with prolonged diarrhoea and after complicated gastrointestinal surgery (Heird and Winters, 1975). In Europe Intralipid ${ }^{\star}$, a fat emulsion, has been widely used as one of the parenteral constituents; its major advantage being that a relatively large amount of calories is supplied in a small volume of fluid. The purpose of this article is to report on the pathological findings of deposition of pigmented material in the reticuloendothelial system in 2 patients who received Intralipid and to consider the possible relation of this to an increased liability to infection.

\section{Case reports}

Case 1. A male was the second child of an Arab family whose parents were first cousins. His elder brother died a year earlier at the age of 3 months at another hospital. The diagnosis was prolonged infantile diarrhoea, seborrhoeic dermatitis, and septicaemia. He was first admitted to our hospital aged 3 months because of recurrent bouts of diarrhoea from the age of 6 weeks and failure to thrive. Physical examination showed mild seborrhoeic dermatitis, diffuse enlargement of all the lymph glands, monilial infection of the finger nails, and bilateral otitis media. He passed 6-10 stools daily. No reducing substances were present in the stools and cultures were negative. His haemoglobin was $10 \mathrm{~g} / \mathrm{dl}$, the leucocyte count and morphology were normal. He was treated with ampicillin for the ear infection, intravenous fluid, and Nutramigen. $f$

Received 14 August 1975.

*Vitrum, Stockholm, Sweden.

†Nutramigen Powder, Mead-Johnson.
An immunodeficiency syndrome was suspected and investigations included serum albumin $4.3 \mathrm{~g} / 100 \mathrm{ml}$, serum globulin $2.4 \mathrm{~g} / 100 \mathrm{ml}, 1 \mathrm{gG} 450 \mathrm{mg} / 100 \mathrm{ml}$, IgA $28 \mathrm{mg} / 100 \mathrm{ml}$, IgM $30 \mathrm{mg} / 100 \mathrm{ml}$. Phytohaemagglutinin blastogenic response of lymphocytes was normal. Serum complement, C3 $115 \mathrm{mg} / 100 \mathrm{ml}$, C4 $20 \mathrm{mg} / 100 \mathrm{ml}$, and total haemolytic complement 55 units. Delayed skin hypersensitivity to Candida antigen was positive. The most likely diagnosis considered was Leiner's disease, but we were unable to perform the specific phagocytic test to prove it. Nevertheless, he was treated throughout with frequent fresh plasma infusions (Miller and Nilsson, 1970).

Two weeks after admission, because of no progress and persistent diarrhoea he was treated with our regimen of parenteral hyperalimentation. This included a daily prepared solution of Amigen, glucose, vitamins, and blood and plasma infusions. $20 \%$ Intralipid (15 ml/kg) was supplemented daily. Trace metals and vitamins were administered according to a standard regimen. The continuous infusion was maintained in the peripheral veins for 30 days. Attempts to transfer to oral feeding during this period failed and a central jugular venous catheter was inserted. There was a sudden deterioration 24 hours later and he died of cardiorespiratory failure.

Necropsy showed that the cause of death was perforation of the catheter into the pleural space with accumulation of fluid. There were no gross changes in the large or small bowel. Many histiocytes were present in the peripheral and medullary sinuses of the lymph nodes; both the thymic-dependent and thymicindependent areas were normal. Normal Hassalls corpuscles were identified in the thymus. The Kupffer cells of the liver contained a diffuse deposition of dark brown coarse pigment (haematoxylin and eosin) (Fig.). 


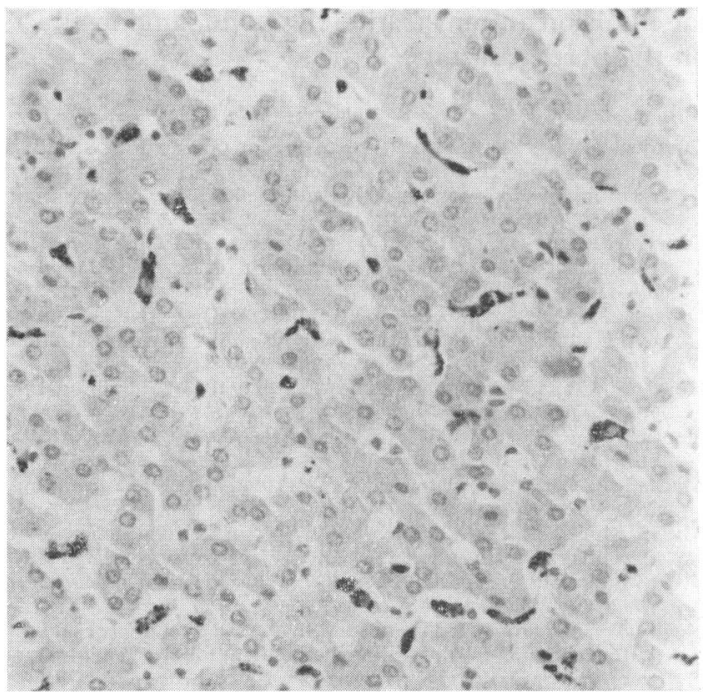

FIG.-Case 1. Liver showing diffuse deposition of pigment in the Kupffer cells. (H. and $E . \times 180$.)

The hepatocytes were uninvolved and no granulomata were found. These pigmented granules were also seen in macrophages of the spleen, lungs, bone marrow, lymph nodes, and thymus. The histochemical characteristics of the pigment in paraffin sections are summarized in the Table. In addition, the pigment was

\section{TABLE}

Histochemical properties of pigment in the Kupffer cells

\begin{tabular}{l|l}
\hline \multicolumn{1}{c|}{ Stain } & \multicolumn{1}{c}{ Reactivity } \\
\hline Haematoxylin-eosin & Dark brown \\
Acid fast (Carbol Fuchsin) & Golden brown \\
Sudan black & Brown-black \\
Masson-Fontana & Brown-black \\
Iron (Perls's Prussian blue) & Brown-yellow \\
P.A.S. & Yellow-orange \\
Ultraviolet fluorescence & Negative \\
Oil red O & Dark brown \\
Bleaching & Yellow-brown \\
& \\
\hline
\end{tabular}

${ }^{\star}$ Most of the granules disappeared with the bleaching stain.

birefringent when viewed under polarized light. A Perls's Prussian blue stain for iron was negative. An interesting finding was coarse vacuolization of the epithelial cells of the proximal and distal convoluted tubules. This finding was considered compatible with the lesion seen after prolonged hyperosmolar fluid administration (Heptinstall, 1966).

Case 2. A male, the third child of healthy unrelated Jewish parents, was born after 36 weeks of gestation and weighed $2 \cdot 35 \mathrm{~kg}$. He was admitted at the age of 6 weeks because of failure to thrive and diarrhoea. Physical examination showed a dystrophic infant and bilateral otitis media. No reducing substances were present in the stool. He was treated with penicillin for the otitis media and several dietary regimens. Because of the persistent diarrhoea and failure of initial therapy, parenteral hyperalimentation was started and was maintained throughout by peripheral venous infusion. $20 \%$ Intralipid $(15 \mathrm{ml} / \mathrm{kg})$ was supplemented daily. Cultures of pus from the ears after paracentesis grew Pseudomonas aeruginosa and Staphylococcus aureus. He was treated with lincomycin and gentamicin and a steady improvement on parenteral hyperalimentation was noted for 15 days. However, at this stage the infant became pyrexial, pale, and apathetic, and septicaemia was suspected. Several blood and urine cultures were negative. Disseminated intravascular coagulation was confirmed by a thrombocyte count of $7000 / \mathrm{mm}^{3}$, a prolonged prothrombin and partial thromboplastin time, and increased fibrinogen split products. No bleeding occurred. Despite appropriate antibiotic therapy and heparin the child died 2 days later.

Necropsy showed multiple large colonies of monilia in the kidneys, myocardium, liver, lungs, bone marrow, and brain. There was minimal infiltration of inflammatory cells around these colonies. Culture of several organs identified the fungus as Candida albicans. The identical pigmented material seen in Case 1 was widely distributed in all the macrophages of the reticuloendothelial system. Histochemical staining was the same as for Case 1.

\section{Discussion}

One of the major hazards of intravenous hyperalimentation is the increased incidence of infection, especially fungal sepsis (Curry and Quie, 1971). Measures to reduce external invasion of organisms, such as the use of a millipore filter, asepsis, and sterilized prepared solutions, are important. Despite this, these patients are still prone to infection. A single experimental study has shown that a defect in host function, namely that of leucocyte opsonic and chemotactic ability, which was related to hypophosphataemia, occurred in parenteral hyperalimentation (Craddock et al., 1974).

Experimental studies have shown that administration of intravenous fat emulsions resulted in the accumulation of pigmented material in the reticuloendothelial system (Neglia et al., 1963). The staining characteristics and morphological appearance of this pigment was similar to that seen in our 2 patients. These findings have not been previously reported to our knowledge in human subjects. It was considered unlikely that the pigment accumulation in our 2 patients occurred from an inherent disease. Firstly, these findings do not conform to any known storage or immunodeficiency disease. In addition, identical findings have been 
noted in an adult patient who received prolonged intravenous feeding including Intralipid after major gastrointestinal surgery (R. David, personal communication). The deposition of material was probably related to the duration and amount of administered Intralipid, as shown in the animal experiments. The occurrence of this pigment was independent of the type of unsaturated fat used and of the presence of emulsifying agents (Thompson et al., 1960). Factors responsible for this pigment accumulation are unclear. It has been suggested that consequent to intravascular haemolysis, haem pigments may catalyse oxidation of unsaturated fatty acids and result in the formation of pigmented lipoprotein complexes which are taken up by the macrophages (Neglia et al., 1963).

The macrophages of the reticuloendothelial system are important 'helper' cells in both the normal humoral and cellular immune response, playing a major role in the resistance to fungal infection together with sensitized T-cells (Mackaness and Blanden, 1967). Several experimental studies have shown that intravenous injection of fat emulsions results in blockade of the reticuloendothelial system, usually with resultant depression of several immune functions. There may be an early stage when stimulation occurs, but eventually depression of function occurs (Di Luzio and Wooles, 1964), especially in prolonged administration.

Our patients showed diffuse deposition of a coarse pigmented material in the macrophages of the reticuloendothelial system after infusion of a fat emulsion. Though no laboratory evidence of the effect of this was shown, it is likely that prolonged fat emulsion administration has wide ranging effects on the host's resistance.

\section{REFERENCES}

Craddock, P. R., Yawata, Y., VanSanten, L., Gilberstadt, S., Silvis, S., and Jacob, H. S. (1974). Acquired phagocyte dysfunction resulting from parenteral hyperalimentation. New England Fournal of Medicine, 290, 1403.

Curry, C. R., and Quie, P. F. (1971). Fungal septicemia in patients receiving pare teral hyperalimentation. New England fournal of Medicine, 285, 1221.

Di Luzio, N. R., and Wooles, W. R. (1964). Depression of phagocytic activity and immune response by methyl palmitate. American Fournal of Physiology, 206, 939.

Heird, W. C., and Winters, R. W. (1975). Total parenteral nutrition. fournal of Pediatrics, 86, 2.

Heptinstall, R. H. (1966). Pathology of the Kidney, p. 679. Churchill, London.

Mackaness, G. B., and Blanden, R. V. (1967). Cellular immunity. Progress in Allergy, 11, 89.

Miller, M. E., and Nilsson, U. R. (1970). A familial deficiency of the phagocytosis-enhancing activity of serum related to a dysfunction of complement $\left(\mathrm{C}_{5}\right)$. New England fournal of Medicine, 282, 354.

Neglia. W., Burrows, L., Thompson, S. W., and Schaffner, F. (1963). Ultrastructural studies of hepatic pigment following administration of intravenous fat. Laboratory Investigation, 12, 378.

Thompson, S. W., Fox, M. A., Forbes, A. L., and Thomassen, R. W. (1960). Residual pigment associated with intravenous fat alimentation. American fournal of Pathology, 36, 355.

Correspondence to Dr. J. H. Passwell, Department of Paediatrics 'B', Chaim Sheba Medical Centre, TelHashomer, Israel.

\section{Addendum}

Since submission of this report, similar findings of pigment deposition have been reported in children after intralipid therapy when administered as part of a parental hyperalimentation.

\section{REFERENCE}

Koga, Y., Swanson, V. L., and Hays, D. M. (1975). Fournal of Pediatric Surgery, 10, 641. 